\section{Covid-19: Anaesthesia procedures may generate fewer aerosols than presumed, study suggests}

\author{
Elisabeth Mahase
}

Anaesthesia procedures such as intubation and extubation during surgery may produce less aerosols than previously thought, according to a small study which is calling for a reappraisal of what constitutes an aerosol generating procedure (AGP) and the associated precautions for routine anaesthetic airway management. ${ }^{1}$

Researchers from North Bristol NHS Trust and the University of Bristol carried out real time, high resolution aerosols monitoring in ultraclean ventilation operating theatres during anaesthesia procedures for insertion (19 recordings) and removal of the tube from the patient's airway (14 recordings).

They reported that these procedures may only produce a fraction of the aerosols previously thought, much less than would be produced during a single regular cough. The authors noted that none of the recordings were from patients known to have covid-19.

During the pandemic, intubation and extubation have been classified as AGPs, meaning that they are believed to produce a fine mist of small particles which could spread the virus to nearby staff. As such, those carrying out these procedures must wear respirators and high level personal protective equipment. After they are carried out, surgery must also be stopped while the operating room is cleared of aerosols and special cleaning is undertaken.

The researchers said these requirements have dramatically slowed surgery and contributed to enormous waiting lists for surgery in the NHS, as well as similar problems in hospitals worldwide. But despite the presumed risk, they claim that no direct measurements of aerosols have ever been made during anaesthetic care in a hospital.

The study, published in Anaesthesia, included recordings from a three week period of surgery for orthopaedic trauma and neurosurgical emergencies. All patients had a controlled anaesthetic induction that included a neuromuscular blocking drug.

The team used the quantity and concentration of aerosolised particles generated by volitional coughs as a reference. They reported that both intubation and extubation sequences produced less aerosol than voluntary coughing.

The average number of particles detected in a five minute period during anaesthetic induction and intubation was seven, compared with a background in the empty theatre of around two particles. The average concentration of particles recorded during the intubation period was 500 times lower than the average concentration recorded during volitional coughs.
Similarly, the maximum concentration recorded during intubation averaged across events was 22 times lower than that seen with volitional coughs, the paper reported.

"For the sequence of tracheal intubation, in particular, the concentration of aerosol generated is several orders of magnitude less than a single cough and is only very modestly above background levels of circulating particles in an ultraclean theatre. These findings demonstrate that the process of tracheal intubation is associated with a very low risk of aerosol generation," the paper said.

"Standard anaesthetic induction and intubation sequences are designed to obtund airway reflexes and the use of a neuromuscular blocking drug ensures that the anaesthetised patient can neither breathe nor cough. Of note we detected no increases in aerosolised particles above the patient's face during anaesthesia, face mask ventilation, airway suction, and, on occasion, several repeated attempts by anaesthetists with a range of experience, providing further reassurance regarding the low level of aerosol generation," it continued.

However, during the tracheal extubation sequence, aerosol concentration was greater than that seen with intubation, with a total number of expectorated airborne particles over a five minute period being similar to a single volitional cough. The paper said a cough event was noted clinically in $50 \%$ of extubations and was frequently detected as an aerosol spike.

"These extubation coughs produced a similar particle size distribution but there were fewer airborne particles than with volitional coughs (around 25\%). Extubation cough aerosol was also transient and only detectable for approximately five seconds," it said. "Mitigation strategies should be considered to reduce the risk of coughing and exposure to aerosols."

Royal College of Anaesthetists vice president William Harrop-Griffiths said the current precautions that protect healthcare workers against infection by aerosol transmission take up a large amount of time and thereby decrease the number of patients that can be cared for in operating theatres.

"If manual lung ventilation and tracheal intubation are not deemed to be AGPs, this saves time, speeds theatre, and greatly increases the NHS's ability to deliver planned surgical care," he said.

Limitations of the study included the relatively small number of observations. The researchers also noted that they did not have control over the specific at intubation. This reflects typical clinical practice up the delivery of care to patients in the operating 
anaesthetic administered or the grade of practitioner, and that the reference coughs were from a single subject (one of the investigators). Additionally, they made no measurements from subjects known to have covid-19 or other intercurrent respiratory comorbidity.

1 Brown J, Gregson FKA, Shrimpton A, etal. A quantitative evaluation of aerosol generation during tracheal intubation and extubation. Anaesthesia 2020. https://associationofanaesthetists-publications.onlinelibrary.wiley.com/doi/10.1111/anae.15292. doi: 10.1111/anae.15292 pmid: 33022093

This article is made freely available for use in accordance with BMJ's website terms and conditions for the duration of the covid-19 pandemic or until otherwise determined by BMJ. You may use, download and print the article for any lawful, non-commercial purpose (including text and data mining) provided that all copyright notices and trade marks are retained. 